\title{
Comparative study of the antioxidant and antibacterial activities of Rumex abyssinicus with commercially available Zingiber officinale and Curcuma longa in Bahir Dar city, Ethiopia
}

Alemayehu Mekonnen ${ }^{1 *}$ (i) and Welday Desta ${ }^{2}$

\begin{abstract}
Background: Rumex abyssinicus (RA) is one of indigenous plants to Ethiopia having traditional values during butter refinement in rural areas. This paper presents a comparative study of the antioxidant and antibacterial activities of RA sample extract to that of Zingiber officinale (ZO) and Curcuma longa (CL).

Methods: Phytochemical screening tests were, first, done using different confirmatory tests prior to quantitative determinations. Quantitative determinations were then conducted - the total phenolic content by the Prussian blue method, total flavonoid content by the aluminum colorimetric assay, and antioxidant activity by the reducing power assay and the cyclic voltammetry technique.

Results: All of the samples showed positive tests, with different intensities, for the presence of phenolics, flavonoids, glycosides and tannins, but not for alkaloids. The total phenolic content, the total flavonoid content and the antioxidant activities of RA (in mg/100 g dry weight) were found to be 319.208 $\pm 15.997,113.252 \pm 1.702$ and 45.632 \pm 2.026 , respectively. On the other hand, both oxidation and reduction peaks were observed in the cyclic voltammetry analysis of the herbs, showing a quasi-reversible redox process of their phenolic compounds. The determination of the inhibition zones (in mm) of ethanol extract of RA was found to be $20.33 \pm 0.58,21.67 \pm 0.58,19.17 \pm 0.29,18.17 \pm 0.29$, and $21.67 \pm 0.58$ against Staphylococcus aureus, MRSA, Streptococcus pneumonia, Escherichia coli, and Shigella flexneri bacteria strains, respectively.

Conclusion: The substantial antioxidant and antibacterial activities of RA compared to $\mathrm{ZO}$ and $\mathrm{CL}$ indicate that the traditional use of the herb to refine butter and keep it safe longer without rancidity formation and other traditional medicinal practices can be attributed to both its antioxidant and antibacterial activities.
\end{abstract}

Keywords: Phytochemical screening, Antioxidant activity, Antibacterial activity, Rumex abyssinicus, Zingiber officinale, Curcuma longa

\footnotetext{
*Correspondence: yirsawnegaalex@gmail.com

${ }^{1}$ Department Chemistry, Science College, Bahir Dar University, P.O. Box:

79, Bahir Dar, Ethiopia

Full list of author information is available at the end of the article
} in this article are included in the article's Creative Commons licence, unless indicated otherwise in a credit line to the material. If material is not included in the article's Creative Commons licence and your intended use is not permitted by statutory regulation or exceeds the permitted use, you will need to obtain permission directly from the copyright holder. To view a copy of this licence, visit http://creativeco mmons.org/licenses/by/4.0/. The Creative Commons Public Domain Dedication waiver (http://creativecommons.org/publicdomain/ zero/1.0/) applies to the data made available in this article, unless otherwise stated in a credit line to the data. 


\section{Background}

Almost all life on Earth is directly or indirectly dependent on plants. Human being is not an exception. It can be dared to say that throughout their ages, human beings have been dependent on plants for their basic needs. Among the wide-range applications of plants, the use of their bioactive components to treat different diseases has been in practice, both traditionally and scientifically [1].

Nowadays, environmental modifications, due to industrialization processes, which result in emission of reactive species, are believed to be the causes of many chronic diseases. Some of these ailments such as cancer are by far known to be caused by a condition called oxidative stress. Oxidative stress is caused by the imbalance of natural defense antioxidants and reactive oxygen species (ROS), and other radicals in the human body $[1,2]$.

Since the last few decades, the potential role of plantderived bioactive substances in alleviating oxidative stress has been extensively studied, and currently phenolic phytochemicals are identified to play this role due to their structural suitability to serve as antioxidants. Antioxidants are those substances that neutralize ROS and other radicals, immediately after their formation [3, 4].

There are many plants that serve as sources of phenolic compounds, among which are herbs and spices. Two of the many herbs and spices that have got worldwide attention since antiquity are Zingiber officinale (ZO) and Curcuma longa (CL), which are commonly known as ginger and turmeric, respectively. Literature data have suggested that $\mathrm{ZO}$ and CL possess high antioxidant activity $[5,6]$.

Despite the wide remedial potential presented by Rumex abyssinicus in Ethiopia, studies on the phytochemical report, antioxidant and antimicrobial activities of this plant are still lacking. It is reported that the herb has been used by Ethiopian traditional practitioners to treat ailments such as mild diabetes, gonorrhea, poisoning, hepatitis, constipation and hypertension [7]. It is also used to treat animals with blackleg and scabies. Bruised eye can be treated by drinking a solution prepared by boiling the roots with butter. The leaf is used for gonorrhea and abscesses. Root of RA plant can preserve skin without structural modification. The tender shoots and leaves of RA are edible and widely used as a vegetable. The stem is chewed like sugar cane for its sweetness and the leaves are eaten as an acidic snack by herdsmen, farmers and children [8]. They have an acid taste and are eaten fresh or cooked, alone or together with other vegetables. The rhizomes yield a yellow and red dye. The dye is used in butter as a condiment, to give it a rich yellow color and as protection against rancidity. The dye is also used to impart a red color to the feet and hands of women [7, 8].
Furthermore, RA is widely used to refine butter in rural Ethiopia. Moreover, it protects butter from spoilage, showing the ability of the plant to prevent rancidity formation. Such capability of the herb can be attributed to antioxidant or/and antimicrobial activities. Although there are some works that show the antimicrobial activity of RA [8], its antioxidant activity is not yet investigated. Therefore, the study of chemical and biological capacities of the plant is unexploited research area in the case of Ethiopia and this can be observed from the limited literature data available.

The use of RA, however, is limited to some parts of the country, and is confined to some traditional practices though it can have spectacular health effects if used properly. Thus, in order to provide scientific justification for the traditional uses of the herb and to show its relative status to the research community, we initiated to focus on the study of phytochemicals, antioxidant and antibacterial activities of RA compared with those of $\mathrm{ZO}$ and CL. We chose $\mathrm{ZO}$ and $\mathrm{CL}$ for comparison since RA is mostly used by the indigenous community for similar purposes. In addition, chemical and biological profiles of $\mathrm{ZO}$ and $\mathrm{CL}$ are extensively studied and sufficient data are available $[9,10]$. This will make our comparison more reliable. Finally, $\mathrm{ZO}$ and $\mathrm{CL}$ are commercially available herbs as food supplement to consumers in the form of either simple, teas, infusions, decoctions, syrup, poultice or standardized extracts worldwide $[9,10]$.

\section{Materials and methods Chemicals and reagents}

Sodium phosphate monobasic dihydrate, sodium phosphate dibasic, potassium ferricyanide, trichloroacetic acid, ferric chloride, gallic acid, ascorbic acid, sodium hydroxide, aluminum chloride, sodium nitrite, ammonia, hydrochloric acid, sulfuric acid, mercuric chloride, potassium iodide, barium chloride dihydrate, sodium chloride, ethanol, chloroform, Muller-Hinton agar powder, all of which are products of Blulux Laboratories (p)Ltd, India, and quercetin (Across organic, New Jersey, USA).

\section{Sample collection and preparation}

Rhizomes of the samples (RA, ZO and CL) with no physical damage were obtained from the main local market around Abay and Tana commercial center in Bahir Dar city, Ethiopia. Each sample was collected from five retailers in order to nullify the differences that may be caused due to handling and storage. The rhizomes of each sample (RA, $\mathrm{ZO}$ and $\mathrm{CL}$ ) were washed with distilled water to remove dirt, and dried at $50{ }^{\circ} \mathrm{C}$ in an air dryer oven. Then, the dried rhizomes were 
first crushed into pieces by mortar and pestle, and then pulverized into powder by an electronic mixer grinder (Fig. 1). The powder of each sample was stored in glass flasks wrapped in an aluminum foil and placed in a dark space until further use.

\section{Extraction of samples}

In all the experiments, fresh extracts were used, where distilled water (as per the cooking conditions) was used as a solvent to extract samples for phytochemical screening and antioxidant activity related tests. Accordingly, for phytochemical screening tests, $1.0 \mathrm{~g}$ powder of each sample was extracted by $100.0 \mathrm{~mL}$ solvent. After cooling to room temperature, the crude content was centrifuged at $3000 \mathrm{rpm}$ for $10 \mathrm{~min}$. Then, it was filtered using Whatman №1 filter paper, and the filtrate was reserved for variety of confirmatory tests. On the other hand, for total phenolic content determination and antioxidant activity determination by the reducing power assay, $0.25 \mathrm{~g}$ powder of each sample was extracted by $250.0 \mathrm{~mL}$ and was further treated as for the phytochemical screening. For the total flavonoid content determination and antioxidant activity determination by the cyclic voltammetry technique, however, $1.0 \mathrm{~g}$ powder of each sample was used because $0.25 \mathrm{~g}$ was too small to be sensed.

For the antibacterial activity determination, the solvents chosen for extraction were ethanol as a polar solvent and chloroform as a low-polarity solvent in order to identify the kind of active substance extracted-whether polar or non-polar. Briefly, $50.0 \mathrm{~g}$ of each sample powder was extracted by $200.0 \mathrm{~mL}$ of solvent. Then the mixture was filtered with Whatman №1 filter paper, and the filtrate was placed in an air dryer oven at $40^{\circ} \mathrm{C}$ until a thick crude extract of about $5 \mathrm{~mL}$ volume was left. This crude extract was then diluted with sterilized, distilled water in a 1:1 (v/v) ratio, and reserved until further use in a laminar flow hood to avoid microbial contamination.

\section{Phytochemical screening}

Phytochemical screening tests were performed for all the extracts following published procedures with slight modifications in order to observe the presence/absence of phenolic compounds, flavonoids, alkaloids, glycosides and tannins [11-13]. All these tests were performed in triplicates.

\section{Total phenolic content determination}

The total phenolic content of the samples was determined by the Prussian blue assay according to methods of prior reports [14] with some modifications. The choice of this method was made due to its simplicity to be used with readily available materials and chemicals. $2.0 \mathrm{~mL}$ of each sample extract was taken into a 50 -mL conical flask, and $20.00 \mathrm{~mL}$ of distilled water was added followed by the addition of $3.0 \mathrm{~mL}$ of $0.008 \mathrm{M} \mathrm{K}_{3} \mathrm{Fe}(\mathrm{CN})_{6}$ and $3.0 \mathrm{~mL}$ of $0.10 \mathrm{M} \mathrm{FeCl}_{3}$ in $0.10 \mathrm{M} \mathrm{HCl}$. After $15 \mathrm{~min}$, a formation of green-to-blue color was observed and the absorbance was taken at $700 \mathrm{~nm}$, the wavelength of maximum absorbance of the colored solution. Gallic acid was used as a standard and a blank was prepared by using distilled water instead of the samples and the standard.

\section{Total flavonoid content determination}

The total flavonoid content was determined by the aluminum chloride colorimetric assay [15] with few modifications. Here, $2.00 \mathrm{~mL}$ of the sample extracts was taken for each sample and added to $4.00 \mathrm{~mL}$ of distilled water followed by an immediate addition of $0.30 \mathrm{~mL}$ of $5 \%$ $\mathrm{NaNO}_{2}$ solution. After $5 \mathrm{~min}, 0.30 \mathrm{~mL}$ of $10 \% \mathrm{AlCl}_{3}$ solution was added, and $6 \mathrm{~min}$ later, $2.0 \mathrm{~mL}$ of $1.00 \mathrm{M} \mathrm{NaOH}$ solution was added. Finally, the absorbance was taken at $510 \mathrm{~nm}$. Quercetin was used as a standard and a blank was prepared by using distilled water instead of the samples and the standard.

\section{Antioxidant activity determination by reducing power assay}

The reducing power assay was used according to prior work [16]. Briefly, to $2.00 \mathrm{~mL}$ of each of sample extract, $2.50 \mathrm{~mL}$ of phosphate buffer $(0.20 \mathrm{M}$, $\mathrm{pH}=6.60$ ) was added followed by addition of $2.50 \mathrm{~mL}$ of $1 \% \mathrm{~K}_{3} \mathrm{Fe}(\mathrm{CN})_{6}$. This mixture was incubated at $50{ }^{\circ} \mathrm{C}$ in water bath for $20 \mathrm{~min}$. Then, $2.50 \mathrm{~mL}$ of $10 \%$ trichloroacetic acid was added. Subsequently, $2.50 \mathrm{~mL}$ of this mixture was taken and $2.50 \mathrm{~mL}$ of distilled water was added to it followed by addition of $0.50 \mathrm{~mL} 0.1 \%$ $\mathrm{FeCl}_{3}$. Formation of green-to-blue colored solution was observed and the absorbance of this solution was taken at $700 \mathrm{~nm}$. Ascorbic acid was used as a standard and a blank was prepared by using distilled water instead of the samples and the standard.

\section{Cyclic voltammetry}

The electrochemical nature of the samples was studied by cyclic voltammetry technique using carbon paste electrode as a working electrode. The choice of carbon paste electrode was made as per its ease of preparation from nearby available materials, and it was previously applied in testing antioxidant activity of plant extracts and gave satisfactory results [17, 18]. Briefly, about $20.00 \mathrm{~mL}$ of sample solutions prepared in phosphate buffer composition $(\mathrm{pH}=7.0, M=0.20 \mathrm{M}$ ) was taken into a $25-\mathrm{mL}$ cell and their cyclic voltammograms were obtained by scanning the potential from $-1000 \mathrm{mV}$ 


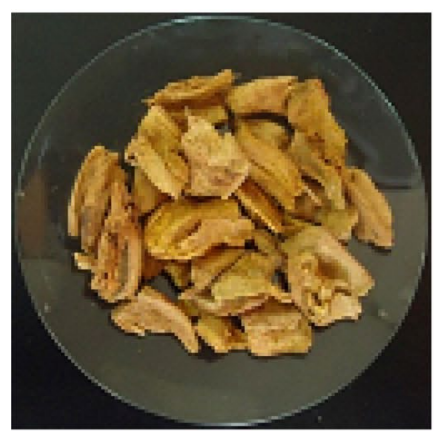

RA

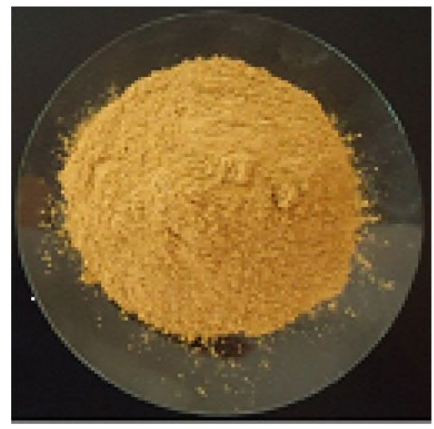

RA

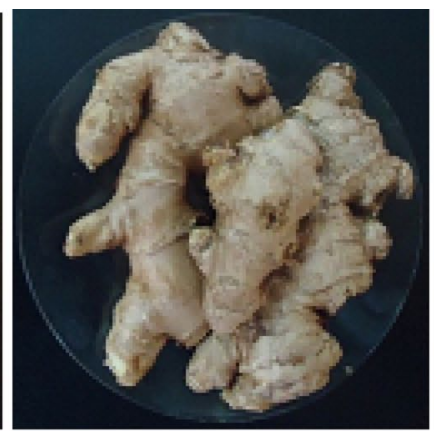

ZO

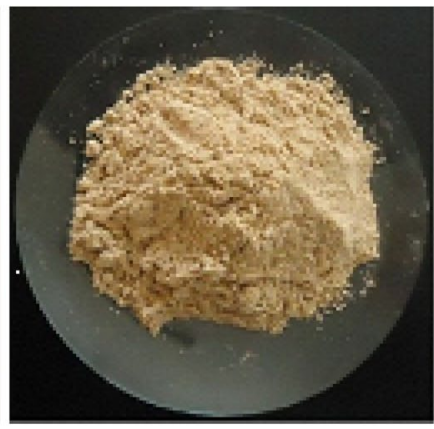

ZO

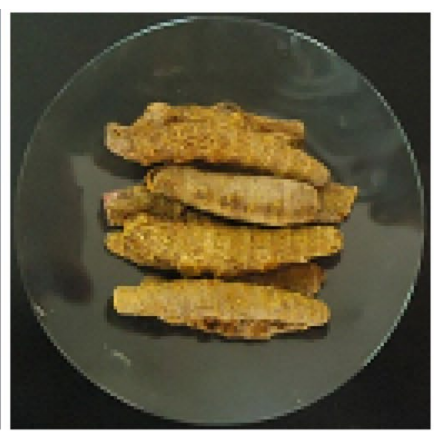

$\mathbf{C}$

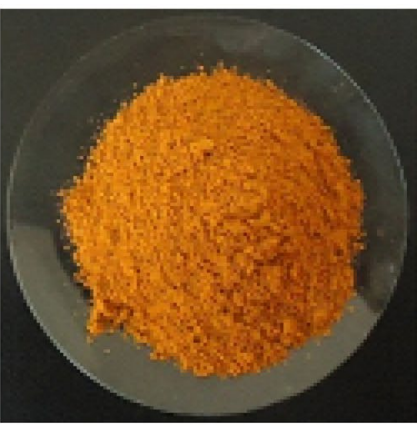

CL

Fig. 1 Rhizomes of dry and powder samples of RA, $Z \mathrm{O}$ and $\mathrm{CL}$, respectively

to $1000 \mathrm{mV}$ at a scan rate of $100 \mathrm{mVs}^{-1}$. Phosphate buffer was used as a blank. All the voltammetric measurements were carried out at room temperature, in a three-electrode configuration. The reference electrode was an $\mathrm{Ag} \mid \mathrm{AgCl} /(\mathrm{KCl}$, saturated) and the counter electrode was a platinum wire. Cyclic voltammetric measurements were performed in a BAS $100 \mathrm{~B}$ electrochemical analyzer connected to a desktop computer and controlled by software (BAS $100 \mathrm{~B}$ ) for windows.

\section{Antibacterial activity determination Test microorganisms}

Microorganisms used included five different bacterial strains, three strains of Gram-positive (Staphylococcus aureus (ATCC 25923), methicillin-resistant Staphylococcus aureus (clinical isolate), and Streptococcus pneumonia (Clinical isolate) and two strains of Gram-negative: Escherichia coli (Clinical isolate), and Shigella flexneri (ATCC 12022). The standard samples were obtained from Department of Biotechnology, University of Gondar and the clinical resistant pathogenic bacteria isolates were collected from Gondar College of Medicine and Health Sciences Hospital. The bacterial cultures were maintained in their appropriate agar slants at $4{ }^{\circ} \mathrm{C}$ until further use.

\section{Susceptibility testing}

The agar well diffusion method was used in the susceptibility testing. Bacterial suspensions of the test microorganisms were made in sterile normal saline and adjusted to the 0.5 McFarland's standard (appropriately prepared from barium chloride dihydrate and sulfuric acid). An inoculum was prepared by seeding the bacterial suspensions in a $100-\mathrm{mL}$ Petri dishes containing freshly prepared Mueller-Hinton agar medium. Then, agar wells were prepared on the inoculum by using a sterilized cork borer with $6 \mathrm{~mm}$ diameter. The wells were $4 \mathrm{~mm}$ deep and about $2.5 \mathrm{~cm}$ apart to minimize overlapping of zones [19]. By using a micropipette, $100 \mu \mathrm{l}$ of the sample extracts, in triplicate, were carefully added to the wells and an antibiotic disc was dispensed with a sterile forceps onto the surface of the inoculated agar plate and pressed down to ensure complete contact with the agar surface. Crude extracts and the antibiotic disc were allowed to diffuse for about $40 \mathrm{~min}$ before incubation and then the plates were incubated in an upright position at $37^{\circ} \mathrm{C}$ for $24 \mathrm{~h}$. Finally, the diameters of inhibition zones (clear transparent regions, including the well diameter) were measured in $\mathrm{mm}$ using a plastic ruler, which was held on the back of the inverted Petri plate and the results were recorded. The antibiotic disc 
[amoxicillin $25 \mu \mathrm{g}$ (AML)] was used as a positive control while sterile, distilled water was used as a negative control.

\section{Statistical method of data analysis}

All measurements were carried out in triplicates $(n=3)$, and values expressed are the mean of three repetitions \pm standard deviation (SD). Results were subjected to analysis of variance (ANOVA) by applying Duncan post hoc test using SPSS 20 software package (IBM Corporation, 1989) and graphs as well as regression coefficients were displayed using Origin 7 software (Origin Lab Corporation, 1991). The differences between means were determined by the least significant difference test, and significance was defined at a confidence limit of $p<0.05$.

In this study, the FRAP assay, total polyphenol and flavonoid contents of the extracts were calculated and reported in terms of ascorbic acid, gallic acid, and quercetin equivalent per gram of extraction. The following analytical Eq. 1 was used for calculation

$$
W\left(\frac{m g}{g o f D W}\right)=\frac{[X(p p m)(D f)(\text { volume of extract }(m l))}{\text { weight of dry sample in gram }}
$$

where $W=\mathrm{AAE}, \mathrm{GE}$ or $\mathrm{QE} X=\frac{Y-C}{\mathrm{Q}}$, dilution factor, $D_{f}=$ $\frac{\text { final volume }}{\text { aliquet volume }}, Y=$ absorbance of sample, $C=y$-intercept from calibration curve and $Q=$ slope from calibration curve.

\section{Results and discussion}

\section{Phytochemical screening}

The presence of phenolic compounds, flavonoids, alkaloids, glycosides and tannins was investigated in the sample extracts using different test methods and the outcomes are tabulated in Table 1. From the table, it can be seen that all the sample extracts showed positive tests for the presence of phenolic compounds, flavonoids, glycosides and tannins except for alkaloids. Moreover, the tests for RA extract showed strongly colored solutions compared to those of $\mathrm{ZO}$ and $\mathrm{CL}$.

\section{Total phenolic content (Prussian blue assay)}

The Prussian blue method is based on the reduction of ferric to ferrous ion by polyphenolic compounds leading to the formation of ferricyanide-ferrous ion complex. The mechanism of the Prussian blue reaction is not yet understood. However, it is thought that removal of a hydrogen atom from the phenolic hydroxyl group is the first step in the reaction [20]. This results in the formation of mesomeric phenoxyl radicals, which may then dimerize or may react with other radicals to form new bonds [21].
Gallic acid standard solutions of concentration 1.2, $30.0,60.0,90.0,120.0$, and $150.0 \mathrm{ppm}$ were used to construct a calibration curve for the determination of the total phenolic content of each sample (RA, ZO, and $\mathrm{CL})$ by the Prussian blue assay. A straight line with an equation $y=0.00323 x+0.0094$ and a linear regression coefficient $\left(R^{2}\right)$ of 0.9997 was obtained, where $y$ is the absorbance, $x$ is the concentration of gallic acid in ppm. Finally, the total polyphenol content of the plant extracts are reported in terms of milligram gallic acid equivalent (mg GAE).

The total phenolic content of the samples were evaluated visually and determined quantitatively. Thus, up on the timely addition of the reagents (potassium hexacyanoferrate and ferric chloride) to each sample extract, the mixture was initially yellow, the color of the reagents. However, few minutes later, the solutions turned light green to deep green and deep blue color showing the differences in the concentration of the total phenolics of each sample. The solutions of some samples did not turn blue (but remained green) due to the probable reason of being masked by the color of the reagents, which were added in excess in order to assure complete oxidation of the polyphenols. However, if the total polyphenol concentration is still high, the formation of blue color can be observed, and was the case for RA. It is estimated that the deepness of the color (green to blue) is directly proportional to the amount of the polyphenol content of samples tested. Hence, as can be seen from Table 2, it can be visually guessed that RA contained the highest total polyphenols followed by $\mathrm{CL}$.

Likewise, CL possesses higher total polyphenol content than that of $\mathrm{ZO}$. The absorbance of all the samples increases with increasing their concentration. This shows the increased ability of their reducing power with increasing concentration, which in turn implies their increment in polyphenol content.

There are considerable differences in the amount of total polyphenol contents of the samples on this study and the literature. Although no data on the polyphenol

\section{Table 1 Results of phytochemical screening experiments}

\begin{tabular}{lccc}
\hline Phytochemicals & \multicolumn{2}{l}{ Samples } & \\
\cline { 2 - 4 } & RA & ZO & CL \\
\hline Phenolics & + & + & + \\
Flavonoids & + & + & + \\
Alkaloids & - & - & - \\
Glycosides & + & + & + \\
Tannins & + & + & + \\
\hline
\end{tabular}

$+=$ positive test, $-=$ absent 
profile of RA could be found, there is inconsistency regarding to the comparison of the total polyphenol content of $\mathrm{ZO}$ and CL. Accordingly, Trinidad et al. [22] found CL to have a significantly greater content of total polyphenols (174 mg GAE/100 g) than that of ZO (55 mg $\mathrm{GAE} / 100 \mathrm{~g}$ ). On the other hand, Maizura et al. [23] found a result of reversed order, indicating $\mathrm{ZO}$ to have higher total polyphenol content (101.6 mg GAE/ $100 \mathrm{~g}$ ) as compared to that of CL (67.9 mg GAE/100 g). Both groups used the Folin-Ciocalteu method to determine the polyphenol contents but in different countries-the Philippines and Malaysia, respectively. The source of differences between the data of this study and the results obtained by the aforementioned researchers might most probably be due to the differences in the methods used as well as the variation in place of origin of the samples. For example, a study conducted in Finland [24] on different herbal spices found that the total polyphenol content of $\mathrm{ZO}$ is $23.5 \mathrm{mg}$ GAE per $\mathrm{g}$ dry weight sample by using the Folin-Ciocalteu method.

\section{Total flavonoid content assay (aluminum chloride colorimetric assay)}

This assay is based on the reaction of flavonoids with aluminum ion at alkaline medium forming colored (pink to red) chelates. When flavonoids are mixed with a $\mathrm{NaNO}_{2}-$ $\mathrm{AlCl}_{3}-\mathrm{NaOH}$ system, there exists a color reaction whose intensity is controlled spectroscopically. The formation of these colored complexes is due to the reaction of flavonoids with the $\mathrm{NaNO}_{2}-\mathrm{AlCl}_{3}-\mathrm{NaOH}$ system such as described by Scheme $1[25,26]$.

Aqueous quercetin standard solutions of concentration 40.0, 100.0, 160.0, 220.0, and $280.0 \mathrm{ppm}$ were used to construct the calibration curve for the determination of the total flavonoid content of each sample by the aluminum chloride colorimetric assay and a straight line with an equation of $y=0.000862 x+0.01833\left(R^{2}=0.9975\right)$ was obtained, where $y$ is the absorbance, $x$ is the concentration of quercetin as the positive control. Then, the total flavonoid content of the samples was calculated in

Table 2 Absorbance of Prussian blue color at different concentrations of samples $(700 \mathrm{~nm})$

\begin{tabular}{llll}
\hline $\begin{array}{l}\text { Concentration } \\
(\% \mathrm{v} / \mathrm{v})\end{array}$ & Absorbance & \\
\cline { 2 - 4 } & RA & ZO & CL \\
\hline 10.00 & $0.119 \pm 0.018$ & $0.038 \pm 0.014$ & $0.095 \pm 0.012$ \\
20.00 & $0.219 \pm 0.017$ & $0.063 \pm 0.010$ & $0.139 \pm 0.010$ \\
30.00 & $0.310 \pm 0.007$ & $0.086 \pm 0.003$ & $0.177 \pm 0.007$ \\
40.00 & $0.402 \pm 0.006$ & $0.110 \pm 0.005$ & $0.224 \pm 0.011$ \\
\hline
\end{tabular}

Data are expressed as mean of three determinations $\pm S D$. There was a significant difference in the mean absorbance of the samples $(p<0.05)$ terms of quercetin (mg QE) per g dry weight of sample. As can be seen from Table 3, the total flavonoid content, in terms of quercetin equivalent, of RA is the highest of all accompanied by that of $\mathrm{ZO}$.

Little information is available on the flavonoid history of RA. Phytochemical screening tests by Teshale and coworkers [7] on aqueous and $80 \%$ methanol extract of the herb indicated that it contains many bioactive constituents among which is flavonoids. Apart from this, no report of quantitative information about the flavonoid content of RA could found. On the other hand, data available on the flavonoid content of $\mathrm{ZO}$ and $\mathrm{CL}$ were not sufficient enough to make comparisons among each other. It is, however, mentioned in one study that CL has higher total flavonoid content than $\mathrm{ZO}$ [22].

\section{Antioxidant capacity assays}

Antioxidant capacity (AOC) assays may be broadly classified as electron transfer (ET)- and hydrogen atom transfer (HAT)-based assays, though in some cases, these two mechanisms may not be differentiated with distinct boundaries because multiple reaction characteristics and mechanisms as well as different phase localizations are usually involved. Thus, no single assay will accurately reflect all of the radical sources or all antioxidants in a mixed or complex system. Clearly, matching radical source and system characteristics to antioxidant reaction mechanisms is critical in the selection of appropriate AOC assay methods [27].

1. Ferric ion reducing antioxidant power (FRAP) assay

The method is based on the reduction of the $\mathrm{Fe}^{3+}$ TPTZ (2,4,6-tripyridyl $s$-triazine) complex to the ferrous form. The antioxidant effect can be evaluated by monitoring the formation of a $\mathrm{Fe}^{2+}-\mathrm{TPTZ}$ complex with a spectrophotometer [28]. The FRAP assay gives fast, reproducible results, but the testing system must be aqueous. Therefore, the reference antioxidant must be a water-soluble one, such as ascorbic acid, uric acid, or Trolox [29].

For the antioxidant activity determination, aqueous solutions of ascorbic acid as reference compound at different concentrations (1.0, 6.0, 11.0, 16.0, 21.0, and $26.0 \mathrm{ppm}$ ) were used to construct the calibration curve by using the reducing power assay and the absorbance was calculated using the equation $y=0.01652 x+0.01715\left(R^{2}=0.9989\right)$, where $y$ is the absorbance, $\mathrm{x}$ is the concentration of ascorbic acid. The antioxidant capacity of the plant extracts are then reported in terms of ascorbic acid (mg AAE) per g dry weight of sample. 


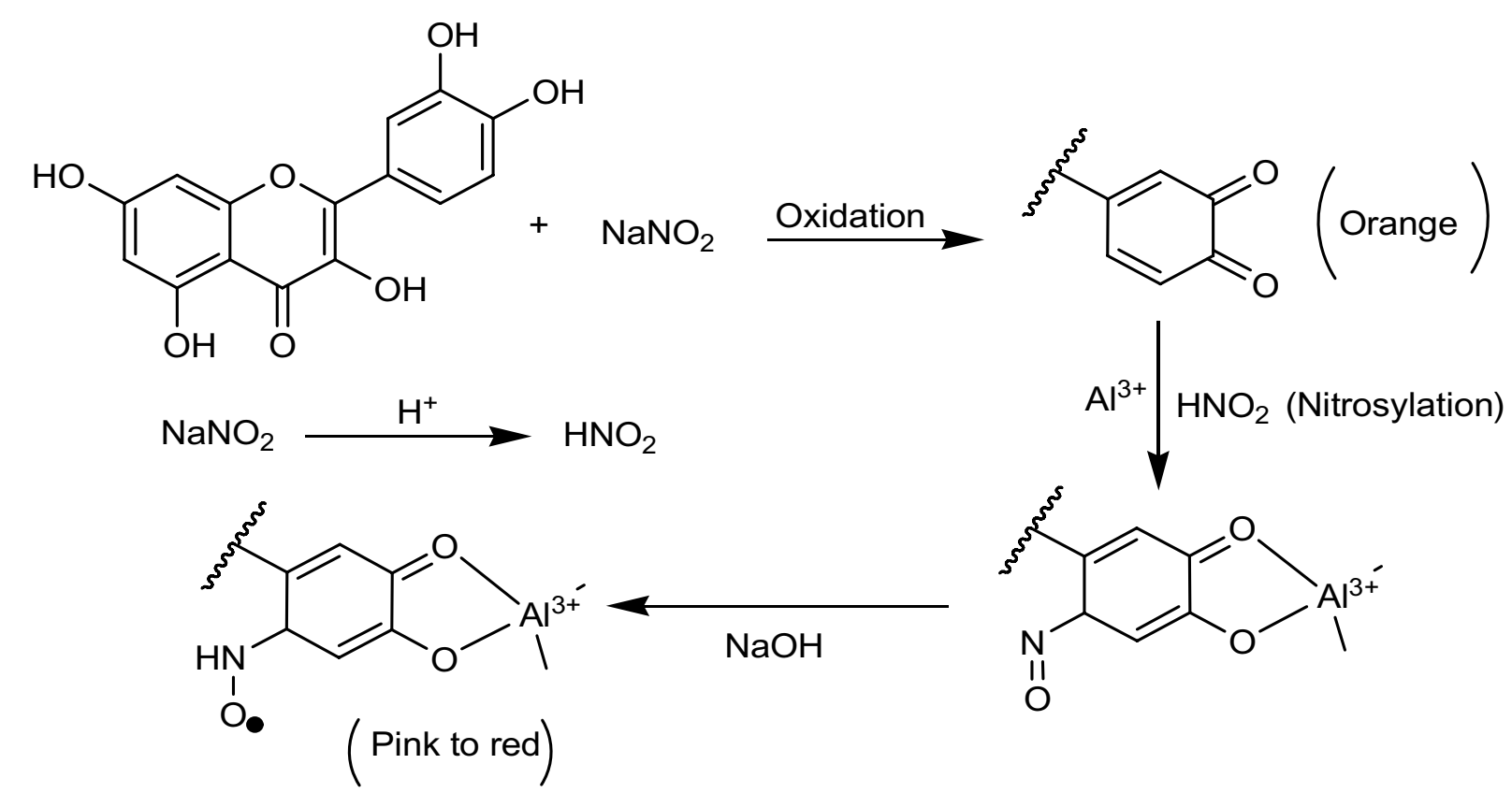

Scheme 1. The color reaction of flavonoids with $\mathrm{Al}^{3+}$

Table 3 Absorbance of $\mathrm{Al}^{3+}$-flavonoid complex at $24 \% \mathrm{v} / \mathrm{v}$ samples of concentration $(510 \mathrm{~nm})$

\begin{tabular}{|c|c|}
\hline Sample $(24 \%, v / v)$ & Absorbance \\
\hline RA & $0.252 \pm 0.004$ \\
\hline $\mathrm{ZO}$ & $0.106 \pm 0.003$ \\
\hline $\mathrm{CL}$ & $0.076 \pm 0.001$ \\
\hline
\end{tabular}

Data are expressed as mean of three determinations \pm SD $(n=3)$. Values in the same column are significantly different $(p<0.05)$ and analyzed by Student's $t$-test

As tabulated in Table 4, it can be inferred that RA again retained its superiority in the antioxidant activity and $\mathrm{ZO}$ is a little bit higher compared to that of $\mathrm{CL}$ in reverse to the order they have in total phenolic content. On the other hand, there is a significant difference in the antioxidant power of RA, ZO, and $\mathrm{CL}$, with RA having the highest of all accompanied by ZO. This difference might be due to the presence of more polyphenols with active hydrogens in the ortho position. Moreover, the Prussian blue assay is very sensitive to groups that have loosely held acidic hydrogens.

At this level, it becomes important to draw a comparison of the total phenolic content, total flavonoid content and the antioxidant activity of the samples in terms of the respective standard equivalents. Figure 2 shows that the reducing power and hence the absorbance of the Prussian blue color of RA significantly increases with increasing concentration, while an increase in concentration of $\mathrm{ZO}$ and $\mathrm{CL}$ causes slight increase. This implies RA has the highest reducing power. This variation can be a result of high polyphenol content, high number of phenolic hydroxyl groups having good structural features that stabilize the resulting radical after electron donation. Here, it is worth noticing that this result of the reducing power for RA is in line with the result obtained for total polyphenol and flavonoid contents where it ranked first. On the other hand, there is a slight difference on the antioxidant activity between $\mathrm{ZO}$ and $\mathrm{CL}, \mathrm{ZO}$ being higher in activity unlike to its order in its polyphenol content. This phenomenon may be due to the presence of non-polyphenolic compounds that contribute to the antioxidant power of $\mathrm{ZO}$.

Such kind of variation might, therefore, be attributed to the structural features of the compound of interest which affects their antioxidant power, as their interactions with antioxidant assays is influenced by the number and position of phenolic hydrogens in their molecules (Scheme 2) [30]. Two of the most suitable structural features of phenolic compounds that characterize their antioxidative potential are the presence of hydrogen-donating substituents (active groups) and the ability to delocalize the resulting free electron in order for it to be stable. The most active com- 
Table 4 Absorbance of test solution at different concentrations of samples in the reducing power assay $(700 \mathrm{~nm})$

\begin{tabular}{llll}
\hline $\begin{array}{l}\text { Concentration } \\
\text { (\%v/v) }\end{array}$ & \multicolumn{2}{l}{ Absorbance } \\
\cline { 2 - 4 } & RA & ZO & CL \\
\hline 24.00 & $0.187 \pm 0.008$ & $0.058 \pm 0.001$ & $0.054 \pm 0.001$ \\
32.00 & $0.259 \pm 0.004$ & $0.073 \pm 0.002$ & $0.066 \pm 0.004$ \\
40.00 & $0.323 \pm 0.004$ & $0.089 \pm 0.002$ & $0.083 \pm 0.004$ \\
48.00 & $0.396 \pm 0.016$ & $0.108 \pm 0.002$ & $0.100 \pm 0.003$
\end{tabular}

Data are expressed as mean of three determinations $\pm S D$. There was a significant difference in the mean absorbance of the samples $(p<0.05)$

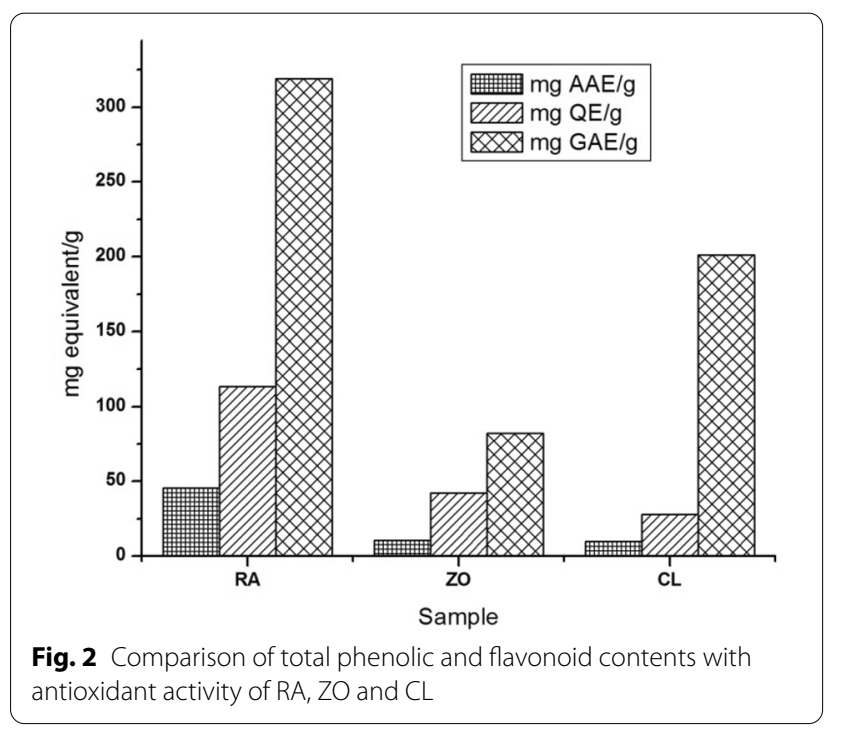

pound as antioxidant is the one possesses more than one active group (e.g., - $\mathrm{OH}$ ) in ortho-position. In other words, the position of the active groups plays an important role of structure-antioxidant relationship activity. The ortho-position was found to be the more active one, due to its ability to form intramolecular hydrogen bonding, followed by para-position and then meta-position of compounds. The $\mathrm{H}$-atom which is not involved in intramolecular hydrogen bonding will then be abstracted by free radicals, resulting in the formation of a stable molecule [31].

This method has been found suitable for the determination of antioxidant capacity of phytochemicals in vitro such as the antioxidant activity of phenolic compounds in different dietary and beverage sources including teas and coffee, citrus fruits and different dry vegetable extracts. The power of cyclic voltammetry results from its ability to rapidly provide considerable information on the thermodynamics of redox processes. The reducing power of a sample is composed of two parameters: the anodic peak potential $\left(E_{\mathrm{Pa}}\right)$ and the anodic peak current $\left(I_{\mathrm{pa}}\right)$ [32]. Correspondingly, the important parameters obtained from a cyclic voltammogram for the oxidizing power of a sample are the intensity of the cathodic peak $I_{\mathrm{Pc}}$ and the cathodic peak potential $\left(E_{P c}\right)$. The reduction potentials of phenolic compounds are direct measure for their ability to undergo oxidation on reaction with oxidizing agents (mostly free radicals). This in turn is dependent on the $\mathrm{O}-\mathrm{H}$ bond dissociation energy (BDE) of the phenolics<smiles>OCCOc1ccccc1O</smiles><smiles>CC1CCCC1C</smiles><smiles>Oc1ccccc1O[CH]c1ccc(I)cc1OI</smiles><smiles>c1ccc2c(c1)O[IH]2</smiles>

Scheme 2. Resonance contributors of polyphenol derivative radicals 
Table 5 Oxidation potentials and peak currents of samples

\begin{tabular}{|c|c|c|c|c|c|c|}
\hline \multirow[t]{2}{*}{ Samples } & \multicolumn{4}{|c|}{ Oxidation } & \multicolumn{2}{|c|}{ Reduction } \\
\hline & $I_{\mathrm{Pa} 1}(\mu \mathrm{A})$ & $E_{\mathrm{Pa} 1}(\mathrm{mV})$ & $I_{\mathrm{Pa} 2}(\mu \mathrm{A})$ & $E_{\mathrm{Pa} 2}(\mathrm{mV})$ & $I_{\mathrm{Pc}}(\mu \mathrm{A})$ & $E_{\mathrm{Pc}}(\mathrm{mV})$ \\
\hline RA & -8.32 & -526.99 & -16.20 & 228.23 & 18.34 & -625.83 \\
\hline ZO & -13.09 & 189.36 & -14.80 & 465.00 & 5.45 & 70.52 \\
\hline$C L$ & -12.65 & 192.00 & - & - & 5.92 & 77.19 \\
\hline
\end{tabular}
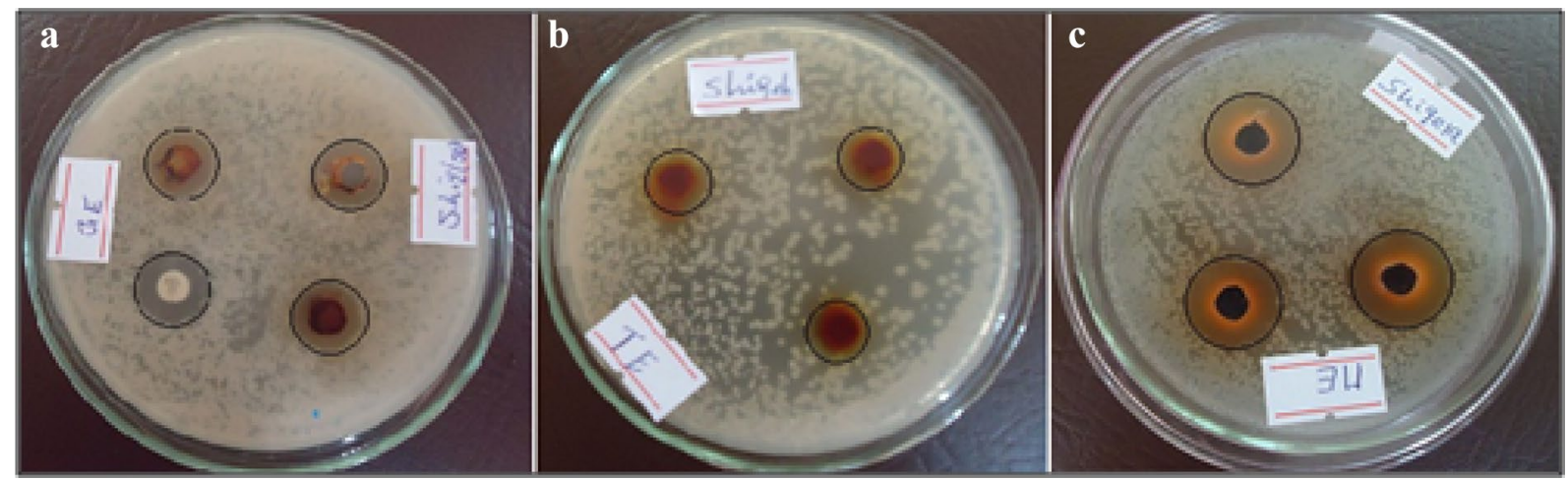

Fig. 3 Inhibition zones (in mm) of a ZO and $A M L$ drug, b CL, and $\mathbf{c}$ RA against Shigella flexneri bacteria

Table 6 Inhibition zones, including well diameter of samples against bacterial growth

\begin{tabular}{|c|c|c|c|c|c|c|}
\hline \multirow[t]{3}{*}{ Solvent } & \multirow{3}{*}{$\begin{array}{l}\text { Samples } \\
\text { and antibiotics }\end{array}$} & \multicolumn{5}{|c|}{ Inhibition zones (in $\mathrm{mm}$ ) for bacteria } \\
\hline & & \multicolumn{3}{|c|}{ Gram-positive } & \multicolumn{2}{|c|}{ Gram-negative } \\
\hline & & S. aureus & MRSA & S. pneumonia & E. coli & S. flexneri \\
\hline \multirow[t]{3}{*}{ Ethanol } & RA & $20.33 \pm 0.58$ & $21.67 \pm 0.58$ & $19.17 \pm 0.29$ & $18.17 \pm 0.29$ & $21.67 \pm 0.58$ \\
\hline & $\mathrm{ZO}$ & $14.33 \pm 0.58$ & $11.83 \pm 0.29$ & $11.83 \pm 0.76$ & $11.00 \pm 1.00$ & $14.00 \pm 0.50$ \\
\hline & $\mathrm{CL}$ & $12.17 \pm 0.29$ & $10.67 \pm 0.58^{b}$ & $10.83 \pm 0.29$ & $10.00 \pm 0.50$ & $11.17 \pm 0.29$ \\
\hline \multirow[t]{3}{*}{ Chloroform } & RA & $11.17 \pm 0.29$ & $13.83 \pm 0.29$ & $7.50 \pm 0.50$ & $7.67 \pm 0.29$ & $11.67 \pm 0.58$ \\
\hline & $\mathrm{ZO}$ & $14.83 \pm 0.29$ & $18.50 \pm 0.50$ & $20.67 \pm 0.58$ & $20.50 \pm 0.50$ & $11.50 \pm 1.32$ \\
\hline & $\mathrm{CL}$ & 0.00 & 0.00 & 0.00 & 0.00 & 0.00 \\
\hline AML & & 14.00 & 12.00 & 10.00 & 11.00 & 14.00 \\
\hline
\end{tabular}

S. aureus Staphylococcus aureus, MRSA methicillin-resistant Staphylococcus aureus, S. pneumonia Streptococcus pneumonia, E. coli Escherichia coli, S. flexneri Shigella flexneri

Each value is the mean $\pm S D$ of three replicates. There is a significant difference in the inhibition zones $(p<0.05)$

[33]. The smaller the $\mathrm{BDE}$, the easier is to remove the hydrogen and the higher is the antioxidant activity of the compound of interest. The ease of oxidizability of phytochemicals is an important measure of their role as antioxidants [34].

Aqueous extracts of the samples (RA, ZO and CL) were subjected to cyclic voltammetry analysis in order to characterize their redox behavior. The electrode potential was linearly varied between two limits ( -1000 to $1000 \mathrm{mV})$ at a specific rate $\left(100 \mathrm{mVs}^{-1}\right)$ while monitoring the current that developed in the electrochemical cell. In order to observe the ease of oxidizability of the sample extracts, the peak parameters obtained from the cyclic voltammograms of each sample are tabulated in Table 5.

2. Antioxidant activity evaluation using cyclic voltammetry

As can be observed from the voltammograms and Table 5, ZO and CL has similar oxidation potentials 
(189.36 and $192.00 \mathrm{mV}$, respectively) and oxidation peak currents $(-13.09$ and $-12.65 \mu \mathrm{A})$, which might be due to the presence of similar chemical constituents in the extracts of both spices. On the other hand, $\mathrm{ZO}$ has an additional peak at $465.00 \mathrm{mV}$ which might be due to the presence of another compound having relatively higher oxidation potential and this can be the reason why $\mathrm{ZO}$ has slightly higher antioxidant activity than CL as can be seen from the voltammograms. Likewise, RA has a positive oxidation potential of $228.23 \mathrm{mV}$, which is slightly higher than the $E_{\mathrm{Pa} 1}$ oxidation potential of $\mathrm{ZO}$ and $\mathrm{CL}$. Moreover, the presence of negative oxidation potential for RA at $-526.99 \mathrm{mV}$ shows the presence of a molecule with higher oxidizability in its extract. On the other hand, all the samples showed reduction peak currents but with different magnitudes relative to the oxidation counterpart. This implies that the redox reaction is not completely reversible but a quasi-reversible one, signifying the potential role of the samples as antioxidants. Again, such situation is associated with the structural feature of the phytochemicals in the plant extracts.

\section{Antibacterial activity determination assay}

A large number of plants are used to combat different diseases and possess antimicrobial activity. In the last few years, a variety of medicinal plants and plant extracts have been screened for their antimicrobial activity. As part of these efforts, many plants have been identified for having different phytochemicals including glycosides, simple phenols and phenolic acids, flavonoids, alkaloids, and terpenoids that are known to possess antimicrobial activities [35, 36].

The antibacterial activity of sample extracts was determined by the agar-well diffusion method. After applying the extract solutions in to the wells of the inoculum and incubating for $24 \mathrm{~h}$ at $37^{\circ} \mathrm{C}$, inhibition zones (clear transparent regions) around the well were observed (Fig. 3).

Significant variation was observed in the antibacterial activities of different extracts. As summarized in Table 6, the result of this study has shown that most of the tested microorganisms were susceptible to both $\mathrm{RA}$ and $\mathrm{ZO}$ extracts and showed a significant inhibition against each bacterium species. It can be seen that ethanol extract of RA showed higher antibacterial activity against all the tested microorganisms than the chloroform correspondent. On the other hand, the chloroform extract of ZO showed a good activity to most of organisms namely methicillinresistant Staphylococcus aureus, Streptococcus pneumonia, and Escherichia coli. However, no inhibition zones were observed for the chloroform extract of CL while it showed relatively smallest inhibition zone in its ethanol extracts.

Thus, ethanol was good enough as solvent to extract the active constituents of RA and $\mathrm{CL}$, while chloroform was better for the extraction of the bioactive constituents of ZO. This implies that the bioactive constituents of RA and $\mathrm{CL}$ are mostly polar ones while that of $\mathrm{ZO}$ are less polar or at least intermediate in polarity.

In conclusion, it is worth noticing at this level that the ethanol extract of RA showed a higher antibacterial effect against all the tested microorganisms compared to the positive control antibiotic drug, AML, and chloroform extract of ZO showed almost better or at least comparable inhibition of bacterial growth in comparison with the positive control.

\section{Conclusion}

The antioxidant investigation of the samples (RA, ZO, and $\mathrm{CL}$ ) showed that RA has a potentially significant activity. It showed the highest total phenolic and flavonoid content as well as antioxidant activity in both the reducing power assay and the cyclic voltammetry technique relative to $\mathrm{ZO}$ and $\mathrm{CL}$. Moreover, ethanol extract of RA showed generally highest antibacterial activity, relative to the aforementioned species, towards all the tested microorganisms. This signifies the presence of more polar kinds of active constituents in the herb. From these results, it can be concluded that RA has the highest potential, in both the antioxidant and antibacterial activity investigations, as compared to $\mathrm{ZO}$ and $\mathrm{CL}$. Thus, the traditional practices of using the rhizome of RA to refine butter and prevent it from rancidity formation can be attributed to both the antioxidant and antibacterial activity of the herb. In general, these results suggest that RA could serve as a good source of natural antioxidants and could potentially be considered as a functional food ingredient.

\section{Abbreviations \\ AOA: Antioxidant activity; GAE: Gallic acid equivalent; QE: Quercetin equiva- lent; TPC: Total phenolic content; TFC: Total flavonoid content; RA: Rumex abys- sinicus; RO: Zingiber officinale; CL: Curcuma longa; MRSA: Methicillin-resistant Staphylococcus aureus; ROS: Reactive oxygen species; AML: Amoxicillin; SD: Standard deviation; ET: Electron transfer; HAT: Hydrogen atom transfer; FRAP: Ferric ion reducing antioxidant power; TPTZ: 2,4,6-Tripyridyl s-triazine; AAE: Ascorbic acid equivalent; $E_{\mathrm{Pa}}$ : Anodic peak potential; $I_{\mathrm{pa}}$ : Anodic peak current; $I_{P C}$ : Cathodic peak current; $E_{P_{C}}$ : Cathodic peak potential; BDE: Bond dissociation energy.}

\section{Acknowledgements}

We are delighted to thank Bahir Dar University for financial support and Dr. Birhanu Andualem for his unreserved support during laboratory work at the Department of Biotechnology in the University of Gondar.

\section{Copyright}

The contents of this manuscript have not been copyrighted or published previously, the contents of this manuscript are not now under consideration for publication elsewhere and the contents of this manuscript will not be copyrighted, submitted, or published elsewhere, while acceptance by the Journal is under consideration. 


\section{Authors' contributions}

AM and WD conceived and designed the experiments: AM analyzed data and wrote the paper. WD performed the experiments. All authors read and approved the final manuscript.

\section{Funding}

Not applicable.

\section{Availability of data and materials}

A herbarium voucher was identified by Dr Ali Seid and deposited in the miniherbarium of a Bahir Dar University, Bahir Dar, Ethiopia. All data generated or analyzed during this study are included in this published article.

\section{Ethics approval and consent to participate}

Not applicable.

\section{Consent for publication}

Not applicable.

\section{Competing interest}

The authors declare no conflict of interest.

\section{Author details}

${ }^{1}$ Department Chemistry, Science College, Bahir Dar University, P.O. Box: 79, Bahir Dar, Ethiopia. ${ }^{2}$ Department of Chemistry, Science College, Samara University, Semera, Ethiopia.

Received: 16 September 2020 Accepted: 22 October 2020

Published online: 11 January 2021

\section{References}

1. Halliwell B, Murcia MA, Chirico S, Aruoma Ol. Free radicals and antioxidants in food and in vivo: what they do and how they work. Crit Rev Food Sci Nutr. 1995;35(I-2):7-20.

2. Bahare S, Shashi U, Ilkay EO, Arun KJ, Daniel AD, Farukh S, Yasaman T, Natália M, Navid B, William CC, Javad S-R. Therapeutic potential of a-and $\beta$-pinene: a miracle gift of nature. Biomolecules. 2019;9(11):738.

3. Bahare S, Miquel M, Jack LA, Antoni S, Natália M, Pawan KM, Mehdi S-R, Pradeep K, Javad S-R. Antioxidants: positive or negative actors? Biomolecules. 2018;8(4):124.

4. Scalbert A, Williamson G. Dietary intake and bioavailability of polyphenols. J Nutr. 2000;130:2073S-2085S

5. Javad S-R, El Youssef R, Alain AR, Carmen S, Raviella Z, Wissam Z, Simona S, Simona R, Katarzyna N-S, Dorota Z, Bahare S, William NS, Noura SD, Yasaman T, El Marc B, Miquel M, Elise AO, Hafiz A, Rasul S, William CC, Alfred M, Natália M. Turmeric and its major compound curcumin on health: bioactive effects and safety profiles for food, pharmaceutical, biotechnological and medicinal applications. Front Pharmacol. 2020;11:01021.

6. Nakatani N. Phenolic antioxidants from herbs and spices. Bio Factors. 2000;13(1-4):141-6.

7. Mekonnen T, Urga K, Engidawork E. Evaluation of the diuretic and analgesic activities of the rhizomes of Rumex abyssinicus Jacq in mice. J Ethnopharmacol. 2010;127(2):433-9.

8. Getie M, Gebre-Mariam T, Rietz R, Hohne C, Huschka C, Schmidtke M, Abate A, Neubert RHH. Evaluation of the antimicrobial and antiinflammatory activities of the medicinal plants Dodonaea viscosa Rumex nervosus and Rumex abyssinicus. Fitoterapia. 2003;74:139-43.

9. Ravindran PN, Babu KN. Ginger: The Genus Zingiber- Medicinal and aromatic plants- industrial profiles. 1st edition, CRC Press. Boca Raton London New York Washington, D.C. 2005; vol. 41

10. Ravindran PN, Babu KN, Sivaraman K. Turmeric: The Genus CurcumaMedicinal and aromatic plants- industrial profiles. 1st edition, CRC Press. Taylor \& Francis Group, LLC, 6000 Broken Sound Parkway NW. 2007; vol. 45

11. Saeed N, Khan MR, Shabbir M. Antioxidant activity, total phenolic and total flavonoid contents of whole plant extracts of Torilis leptophylla L. BMC Complement Alternat Med. 2012;12:221.

12. Senguttuvan J, Paulsamy S, Karthika K. Phytochemical analysis and evaluation of leaf and root parts of the medicinal herb, Hypochaeris radicata L. for in vitro antioxidant activities. Asian Pac J Trop Biomed. 2014;4(1):S359-67.

13. Kähkönen MP, Hopia Al, Vuorela HJ, Rauha JP, Pihlaja K, Kujala TS, Heinonen M. Antioxidant activity of plant extracts containing phenolic compounds. J Agric Food Chem. 1999;47:3954-62.

14. Margraf T, Karnopp AR, Rosso ND, Granato D. Comparison between folin-ciocalteu and prussian blue assays to estimate the total phenolic content of juices and teas using 96-well microplates. J Food Sci. 2015:80(11):C2397-403.

15. Zhishen J, Mengcheng T, Jianming W. The determination of flavonoid contents in mulberry and their scavenging effects on superoxide radicals. Food Chem. 1999;64:555-7.

16. Hinneburg I, Dorman HJD, Hiltunen R. Antioxidant activities of extracts from selected culinary herbs and spices. Food Chem. 2006a;97:122-9.

17. Mekonnen A, Atlabachew M, Kassie B. Investigation of antioxidant and antimicrobial activities of Euclea schimperi leaf extracts. Chem Biol Technol Agric. 2018;5:16-27.

18. Apetrei C, Apetrei IM, De Saja JA, Rodriguez-Mendez ML. Carbon paste electrodes made from different carbonaceous materials: application in the study of antioxidants. Sensors. 2011:11:1328-44.

19. Srinivasan D, Nathan S, Suresh T, Perumalsamy PL. Antimicrobial activity of certain Indian medicinal plants used in folkloric medicine. J Ethnopharmacol. 2001; 174(3): 217-220.

20. Adhikamsetty RK, Jonnalagadda SB. Kinetics and mechanism of Prussian blue formation. Bull Chem Soc Ethiopia. 2009;23(1):47-54.

21. Graham HD. Stabilization of the Prussian blue color in the determination of polyphenols. Agric Food Chem. 1992;40:801-5.

22. Trinidad TP, Sagum RS, De Leon MP, Mallillin AC, Borlagdan MP. Zingiber officinale and Curcuma longa as potential functional foods/ingredients. Food Public Health. 2012;2(2):1-4.

23. Maizura M, Aminah A, Wan Aida WM. Total phenolic content and antioxidant activity of kesum (Polygonum minus), ginger (Zingiber officinale) and turmeric (Curcuma longa) extract. Int Food Res J. 2011;18(2):529-34.

24. Hinneburg I, Dorman HJD, Hiltunen R. Antioxidant activities of extracts from selected culinary herbs and spices. Food Chem. 2006b;97(1):122-9.

25. Grotewold E. The science of flavonoids. Springer; 2006 edition

26. Zhu H, Wang Y, Liu Y, Xia Y, Tang T. Analysis of flavonoids in portulaca oleracea L. by UV-Vis spectrophotometry with comparative study on different extraction technologies. Food Anal Methods. 2010;3:90-7.

27. Prior RL, Wu X, Schaich K. Standardized methods for the determination of antioxidant capacity and phenolics in foods and dietary supplements. J Agric Food Chem. 2005;53(10):4290-302.

28. Huang D, Ou B, Prior RL. The chemistry behind antioxidant capacity assays. J Agric Food Chem. 2005;53:1841-56.

29. Moon J-K, Shibamoto T. Antioxidant Assays for Plant and Food Components. J Agric Food Chem. 2009;57:1655-66

30. Bendary E, Francis RR, Ali HMG, Sarwat MI, Hady SEl. Antioxidant and structure-activity relationships (SARs) of some phenolic and anilines compounds. Ann Agric Sci. 2013;58(2):173-81.

31. Caballero B, Finglas P, Toldra F. Encyclopedia of food sciences and nutrition. 2nd ed. London: Academic Press; 2003. p. 4507-14.

32. Chevion S, Roberts MA, Chevion M. The use of cyclic voltammetry for the evaluation of antioxidant capacity. Free Radical Biol Med. 2000;28(6):860-70.

33. Olszewska MA, Michel P. Antioxidant activity of inflorescences, leaves and fruits of three Sorbus species in relation to their polyphenolic composition. Nat Prod Res. 2009;23(16):1507-21.

34. Adhikari S, Priyadarsini Kl, Mukherjee T. Physicochemical studies on the evaluation of the antioxidant activity of herbal extracts and active principles of some Indian medicinal plants. J Clin Biochem Nutr. 2007;40:174-83.

35. Arora DS, Kaur J. Antimicrobial activity of spices. Int J Antimicrob Agents. 1999;12(3):257-62.

36. Gonzalez-Lamothe R, Mitchell G, Gattuso M, Diarra MS, Malouin F, Bouarab K. Plant antimicrobial agents and their effects on plant and human pathogens. J Mol Sci. 2009;10:3400-19.

\section{Publisher's Note}

Springer Nature remains neutral with regard to jurisdictional claims in published maps and institutional affiliations. 